\title{
Perceptions of epigenetics
}

\author{
Adrian Bird $^{1}$
}

\section{Geneticists study the gene; however, for epigeneticists, there is no obvious 'epigene'. Nevertheless, during the past year, more than 2,500 articles, numerous scientific meetings and a new journal were devoted to the subject of epigenetics. It encompasses some of the most exciting contemporary biology and is portrayed by the popular press as a revolutionary new science - an antidote to the idea that we are hard-wired by our genes. So what is epigenetics?}

There has always been a place in biology for words that have different meanings for different people. Epigenetics is an extreme case, because it has several meanings with independent roots. To Conrad Waddington, it was the study of epigenesis: that is, how genotypes give rise to phenotypes during development ${ }^{1}$. By contrast, Arthur Riggs and colleagues defined epigenetics as "the study of mitotically and/or meiotically heritable changes in gene function that cannot be explained by changes in DNA sequence"2: in other words, inheritance, but not as we know it. These definitions differ markedly, although they are often conflated as though they refer to a single phenomenon. Waddington's term encompasses the activity of all developmental biologists who study how gene activity during development causes the phenotype to emerge, but it suffers from the disadvantage that developmental biologists themselves rarely, if ever, use this word to describe their field. In this sense, the usage is obsolete. The definition put forward by Riggs and colleagues tells us what epigenetics is not (inheritance of mutational changes), leaving open what kinds of mechanism are at work. In this article, I give examples of how epigenetic phenomena are studied and interpreted, and I propose a revised definition that embodies contemporary usage of the word.

The molecular basis of heritable epigenetics has been studied in a variety of organisms. The DNA methylation system and the Polycomb/ Trithorax systems come closest to the ideal, because alterations in these systems are often inherited by subsequent generations of cells and sometimes organisms (Box 1). A classic case of what Robin Holliday named epimutation ${ }^{3}$ is the peloric variant of toadflax (Linaria) flowers (Fig. 1), first described by Linnaeus. In this variant, heritable silencing of the gene $L c y c$, which controls flower symmetry, is due not to a conventional mutation (that is, a mutation in the nucleotide sequence) but to the stable transmission of DNA methylation at this locus from generation to generation ${ }^{4}$. Although most variants arising in laboratory plants are due to conventional mutations rather than epimutations of this kind, examples of transgenerational epigenetics are now well documented in plants (see page 418) and fungi. In animals, however, the transmission of epigenetic traits between organismal generations has, so far, been detectable only by using highly sensitive genetic assays ${ }^{5}$. The mouse agouti locus (also known as nonagouti), which affects coat colour, is the best-studied example, being affected by the extent of DNA methylation at an upstream transposon. Genetically identical parents whose agouti genes are in different epigenetic states tend to produce offspring with different coat colours, although the effect is variable.

Despite the paucity of data from animal studies, this type of epigenetics has caught the general imagination because, in principle, it is stable but potentially affected by the environment. The possibility that acquired 'marks' can be passed from parents to children has a deliciously lamarckian flavour that has proved difficult to resist as a potential antidote to genetic determinism. A recent $\mathrm{BBC}$ television science programme hailed the advent of epigenetics as a profound shift in our understanding of inheritance (http://www.bbc.co.uk/sn/tvradio/programmes/horizon/ ghostgenes.shtml). It summarized the implications of the emergent science as follows: "At the heart of this new field is a simple but contentious idea - that genes have a 'memory'. That the lives of your grandparents - the air they breathed, the food they ate, even the things they saw - can directly affect you, decades later, despite your never experiencing these things yourself." Is there any evidence for these heady claims, and how reliable is it? The answer to the first part of the question is yes.

\section{Genes learning by experience?}

Several studies have reported evidence that links the environment or ageing to long-lasting epigenetic effects on phenotype. One study

\section{Box 1 | Epigenetic paradigms}

There are two classic epigenetic systems: the Polycomb and Trithorax (Polycomb/Trithorax) systems, and DNA methylation. The Polycomb and Trithorax groups of proteins, which are named after mutants of the fruitfly Drosophila melanogaster, work to maintain repressed or active transcription states, respectively, of developmentally important genes. In the absence of these systems, the genes that specify the different segments of the fruitfly are initially expressed correctly, but this pattern cannot be maintained. It can be inferred from this that the Polycomb/ Trithorax systems stably 'memorize' gene-expression patterns that have been set up by other cellular mechanisms. There is evidence that Polycomb-imposed silencing can even be transmitted between fruitfly generations at low frequency ${ }^{18}$. Biochemical studies have enabled the identification of components of the two key Polycomb-system protein complexes and have established a close link with modification of the lysine residue at position 27 of histone $\mathrm{H} 3$. The mechanism by which silencing is transmitted between cell generations remains obscure.

In the case of DNA methylation, biochemical information preceded genetic understanding of the system. The methylated sequence in vertebrates is CG, which is paired with the same sequence on the opposite DNA strand. This symmetry means that sites are transiently methylated on only one of the two DNA strands (that is, hemimethylated) after DNA replication. CG methylation patterns are copied between cell generations by the DNA methyltransferase DNMT1, which 'completes' hemimethylated but not unmethylated sites. In plants and fungi, the base 5-methylcytosine is also present in non-symmetrical DNA sequences, so the mechanism of copying is less obvious. DNA methylation is associated with stable gene silencing (for example, on the inactive $X$ chromosome), either through interference with transcription-factor binding or through the recruitment of repressors that specifically bind sites containing methylated CG. 
examined monozygotic (that is, identical) twins, whom, perhaps oddly, epigeneticists often use to exemplify their system at work. To many, twins epitomize the awesome power of genetics to determine human form and function regardless of environment. Indeed, 'concordance' of a particular characteristic in monozygotic and dizygotic twins is one of the most reliable ways of assessing its genetic basis. What has attracted the attention of epigeneticists, however, is that monozygotic twins do not always show the same disease susceptibility, raising the possibility that epigenetic differences that arise during ageing are at work ${ }^{6}$. Accordingly, it has been reported that young twins have similar amounts of DNA methylation, whereas older twins differ considerably in the amounts and patterns of this modification ${ }^{7}$. Might these non-genetic age-dependent differences in gene marking give rise to the divergent disease predispositions seen in some twins? At present, this is unclear, and a recent study emphasizes the need for further basic work on twins. The largest high-resolution analysis of human DNA methylation patterns so far found that 873 genes on 3 chromosomes showed no significant variation in DNA methylation between individuals in their mid-20s and those in their mid- $60 \mathrm{~s}^{8}$. The remarkable uniformity of DNA methylation among unrelated individuals of disparate ages does not square easily with the large divergence reported in twins of the same age.

Another high-profile study has raised the possibility that a mother's behaviour can affect the chemistry of DNA in her offspring. Quality of early maternal care has long been acknowledged to have long-term repercussions during the lifetime of an individual. A potential mechanism for this effect was deduced from a study reporting that maternal nurturing in rats alters DNA methylation at the gene encoding the glucocorticoid receptor ${ }^{9}$. The authors suggest that in the absence of appropriate nurturing, there is less methylation of this gene in the hippocampus, resulting in overexpression of the receptor in later life. The implication is that the glucocorticoid-mediated stress-response pathway is epigenetically fixed at the level of gene transcription. In addition, transgenerational effects of environmental insults have been reported in mammals: for example, the exposure of embryonic rats to the antiandrogenic compound vinclozolin led to a decrease in spermatogenesis not only in the treated animals but also in males of several subsequent generations ${ }^{10}$. Altered DNA methylation was again suggested as a potential mediator of this effect, although, during development, mammalian embryos pass through a profoundly hypomethylated state, which might be expected to jeopardize the heritability of such marks. Despite uncertainties about the mechanism(s) at work, these studies have raised the profile of epigenetics as a potential mechanistic explanation for the longterm impact of the environment on physiology and behaviour (see page 433). Time will tell whether that potential is realized.

\section{Epigenetics and inheritance}

Should heritability be mandatory in a contemporary view of epigenetics? The requirement that epigenetic characters should be transmissible
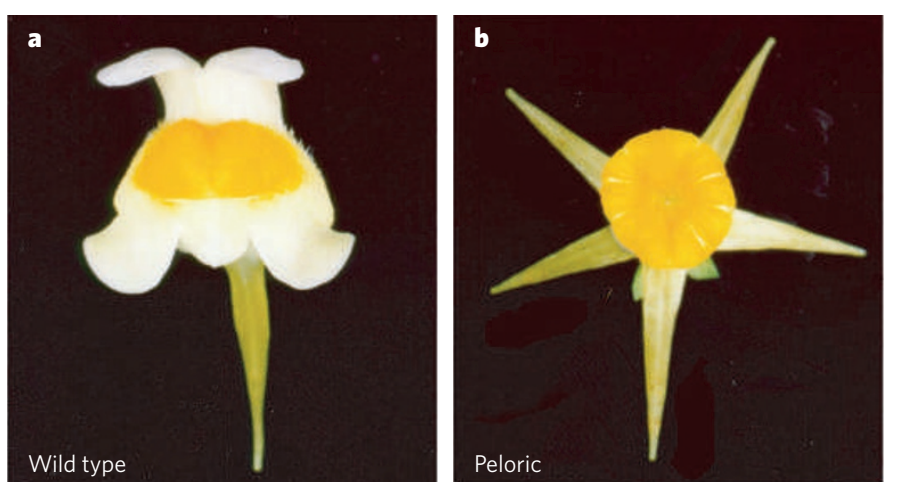

Figure 1 | Frontal view of a wild-type toadflax flower and a peloric epimutant. a, The wild-type flower is dorsoventrally asymmetrical. b, By contrast, the peloric flower is radially symmetrical with all petals resembling the ventral petal of the wild-type flower. (Image reprinted, with permission, from ref. 4.)
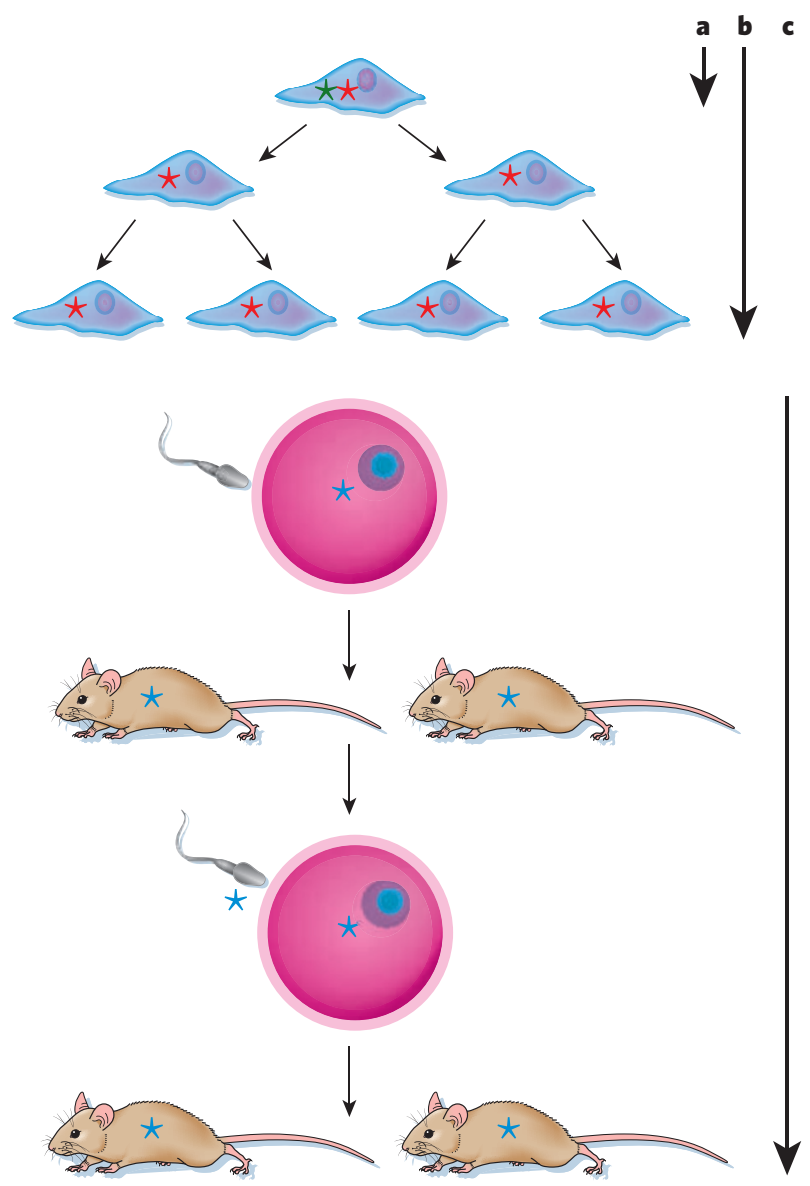

Figure 2 | Persistence of epigenetic marks. Alterations that last less than one cell cycle (green asterisk, a) do not qualify as epigenetic under the definition that strictly requires heritability, whereas non-mutational changes that are transmitted from one cell to its daughters (red asterisk, b) or between generations of an organism (blue asterisk, c) do qualify.

through mitosis or meiosis has the virtue of clarity but can be a liability. To explain why, it is necessary to introduce a third, somewhat informal, 'definition' of epigenetics that has crept into widespread use. This incarnation of epigenetics encompasses the biology of chromatin, including the complex language of chromatin marks (see page 407), the transcriptional effects of RNA interference (see page 399) and, for good measure, the effects of the higher-order structure of chromosomes and the nucleus (see page 413). The attraction of this usage is that it brackets together some of the most exciting contemporary work in biology. Its drawback is that it does not sit easily with the prevailing textbook definitions. One reason for this is that many chromatin marks are short-lived. For example, phosphorylation of the variant histone H2AX (also known as H2AFX) after a double-strand break ${ }^{11}$ would qualify as an epigenetic mark under the emerging definition, but it is too transient to qualify as a heritable epigenetic mark (Fig. 2). Histone modifications associated with transcription are also ambiguous with respect to heritability. On the one hand, DNA methylation affects histone acetylation and histone methylation, so these modifications can be viewed as heritably epigenetic, albeit indirectly ${ }^{12}$. On the other hand, these histone marks can also result from events that seem to involve neither DNA methylation nor Polycomb group proteins, and the marks are not necessarily transmissible between generations. Therefore, a single histone modification could, in principle, be rated as either epigenetic or not epigenetic according to the heritability credentials of its origin. Such a complicated classification system would have limited utility.

The issue of replicative accuracy is also relevant when considering heritability. DNA synthesis is spectacularly accurate, making only 
1 'unforced' error for every $10^{7}-10^{8}$ bases copied ${ }^{13}$. But DNA methylation has an apparent accuracy of $\sim 96 \%$, which is $\sim 1$ error for every 25 methylated sites copied ${ }^{14}$. Because of this error rate, cloning from a single cell quickly results in a population of cells in which DNA methylation patterns are diverse ${ }^{15}$. Methylated domains are more stably maintained, even though the detailed location of methylated sites varies within them. But even the peloric variant of toadflax, which is an otherwise perfect example of heritable epigenetics in action, shows considerable instability as the plant grows. So how accurately transmitted should an epigenetic mark be? Variation due to faulty copying is compounded by current evidence that all histone modifications, as well as DNA methylation itself, can be abruptly removed during development, thereby preventing the persistence of these modifications in a heritable epigenetic sense (see page 425). The restrictiveness of the heritable view of epigenetics is perhaps best illustrated by considering the brain. A growing idea is that functional states of neurons, which can be stable for many years, involve epigenetic phenomena ${ }^{16}$, but these states will not be transmitted to daughter cells because almost all neurons never divide.

\section{Refining a definition}

Given that there are several existing definitions of epigenetics, it might be felt that another is the last thing we need. Conversely, there might be a place for a view of epigenetics that keeps the sense of the prevailing usages but avoids the constraints imposed by stringently requiring heritability. The following could be a unifying definition of epigenetic events: the structural adaptation of chromosomal regions so as to register, signal or perpetuate altered activity states. This definition is inclusive of chromosomal marks, because transient modifications associated with both DNA repair or cell-cycle phases and stable changes maintained across multiple cell generations qualify. It focuses on chromosomes and genes, implicitly excluding potential three-dimensional architectural templating of membrane systems and prions, except when these impinge on chromosome function. Also included is the exciting possibility that epigenetic processes are buffers of genetic variation, pending an epigenetic (or mutational) change of state that leads an identical combination of genes to produce a different developmental outcome ${ }^{17}$.

An implicit feature of this proposed definition is that it portrays epigenetic marks as responsive, not proactive. In other words, epigenetic systems of this kind would not, under normal circumstances, initiate a change of state at a particular locus but would register a change already imposed by other events. Such events could be, for example, the collision of DNA with ionizing radiation or a developmental switch in gene expression. It could be argued that the responsive nature of epigenetic processes is a unifying feature, because classic epigenetic systems such as the DNA methylation system and the Polycomb/Trithorax systems seem to respond to previous switches in gene activity in this way. Therefore, their sophisticated feature is the ability, in the 'darkness' of the nucleus, to sense and mark changes in the chromosomal status. For example, transcriptional activation through sequence-specific DNA-binding proteins brings in histone acetyltransferases, which then epigenetically adapt the promoter region for transcription (for histone acetyl groups, although ephemeral, would now be epigenetic). Similarly, elongating polymerases carry enzymes that restrain the spurious transcriptional initiation that might arise within the temporarily disrupted chromatin of an active gene. Without such epigenetic mechanisms, hard-won changes in genetic programming could be dissipated and lost; transient disruptions of chromosomal organization might go uncompensated; and DNA damage might escape repair.

1. Waddington, C. H. The Strategy of the Genes (Allen \& Unwin, London, 1957).

2. Russo, V. E. A., Martienssen, R. A. \& Riggs, A. D. (eds) Epigenetic Mechanisms of Gene Regulation (Cold Spring Harbor Laboratory Press, Woodbury, 1996).

3. Jeggo, P. A. \& Holliday, R. Azacytidine-induced reactivation of a DNA repair gene in Chinese hamster ovary cells. Mol. Cell. Biol. 6, 2944-2949 (1986).

4. Cubas, P., Vincent, C. \& Coen, E. An epigenetic mutation responsible for natural variation in floral symmetry. Nature 401, 157-161 (1999).

5. Chong, S. \& Whitelaw, E. Epigenetic germline inheritance. Curr. Opin. Genet. Dev. 14, 692-696 (2004).

6. Wong, A. H., Gottesman, I. I. \& Petronis, A. Phenotypic differences in genetically identical organisms: the epigenetic perspective. Hum. Mol. Genet. 14, R11-R18 (2005).

7. Fraga, M. F. et al. Epigenetic differences arise during the lifetime of monozygotic twins. Proc Natl Acad. Sci. USA 102, 10604-10609 (2005).

8. Eckhardt, F. et al. DNA methylation profiling of human chromosomes 6, 20 and 22. Nature Genet. 38, 1378-1385 (2006)

9. Weaver, I. C. et al. Epigenetic programming by maternal behavior. Nature Neurosci. 7, 847-854 (2004).

10. Anway, M. D., Cupp, A. S., Uzumcu, M. \& Skinner, M. K. Epigenetic transgenerational actions of endocrine disruptors and male fertility. Science 308, 1466-1469 (2005).

11. Rogakou, E. P., Boon, C., Redon, C. \& Bonner, W. M. Megabase chromatin domains involved in DNA double-strand breaks in vivo. J. Cell Biol. 146, 905-916 (1999).

12. Klose, R. J. \& Bird, A. P. Genomic DNA methylation: the mark and its mediators. Trends Biochem. Sci. 31, 89-97 (2006).

13. Kunkel, T. A. DNA replication fidelity. J. Biol. Chem. 279, 16895-16898 (2004).

14. Laird, C. D. et al. Hairpin-bisulfite PCR: assessing epigenetic methylation patterns on complementary strands of individual DNA molecules. Proc. Natl Acad. Sci. USA 101, 204-209 (2004).

15. Silva, A. J., Ward, K. \& White, R. Mosaic methylation in clonal tissue. Dev. Biol. 156, 391-398 (1993).

16. Hong, E. J., West, A. E. \& Greenberg, M. E. Transcriptional control of cognitive development. Curr. Opin. Neurobiol. 15, 21-28 (2005)

17. Sollars, V. et al. Evidence for an epigenetic mechanism by which $\mathrm{Hsp} 9 \mathrm{O}$ acts as a capacitor for morphological evolution. Nature Genet. 33, 70-74 (2003).

18. Cavalli, G. \& Paro, R. The Drosophila Fab-7 chromosomal element conveys epigenetic inheritance during mitosis and meiosis. Cell 93, 505-518 (1998).

Acknowledgements I thank the Wellcome Trust for research support.

Author Information Reprints and permissions information is available at npg.nature.com/reprintsandpermissions. The author declares no competing financial interests. Correspondence should be addressed to the author (apbird@staffmail.ed.ac.uk). 